\title{
OPEN Comparison of new implantation of cardiac implantable electronic device between tertiary and non-tertiary hospitals: a Korean nationwide study
}

Seungbong $\mathrm{Han}^{1}$, Gyung-Min Park ${ }^{2}$, Yong-Giun Kim ${ }^{\circledR}$, Ki Won Hwang ${ }^{3}$, Chang Hee Kwon ${ }^{4}$, Jae-Hyung Roh ${ }^{5}$, Sangwoo Park², Ki-Bum Won ${ }^{2}$, Soe Hee Ann², Shin-Jae Kim² \& Sang-Gon Lee ${ }^{2}$

This study compared the characteristics and mortality of new implantation of cardiac implantable electronic device (CIED) between tertiary and non-tertiary hospitals. From national health insurance claims data in Korea, 17,655 patients, who underwent first and new implantation of CIED between 2013 and 2017, were enrolled. Patients were categorized into the tertiary hospital group $(n=11,560)$ and non-tertiary hospital group $(n=6095)$. Clinical outcomes including in-hospital death and all-cause death were compared between the two groups using propensity-score matched analysis. Patients in non-tertiary hospitals were older and had more comorbidities than those in tertiary hospitals. The study population had a mean follow-up of $2.1 \pm 1.2$ years. In the propensity-score matched permanent pacemaker group ( $n=5076$ pairs), the incidence of in-hospital death (odds ratio [OR]: 0.76 , 95\% confidence interval [CI]: $0.43-1.32, p=0.33$ ) and all-cause death (hazard ratio [HR]: $0.92,95 \%$ $\mathrm{Cl} 0.81-1.05, p=0.24$ ) were not significantly different between tertiary and non-tertiary hospitals. These findings were consistently observed in the propensity-score matched implantable cardioverterdefibrillator group $(\mathrm{n}=992$ pairs, OR for in-hospital death: $1.76,95 \% \mathrm{Cl} 0.51-6.02, p=0.37 ; \mathrm{HR}$ for all-cause death: $0.95,95 \% \mathrm{Cl} 0.72-1.24, p=0.70$ ). In patients undergoing first and new implantation of CIED in Korea, mortality was not different between tertiary and non-tertiary hospitals.

The use of cardiac implantable electronic devices (CIEDs), including permanent pacemaker (PPM), cardiac resynchronization therapy with pacemaker (CRT-P) or defibrillator (CRT-D), and implantable cardioverterdefibrillator (ICD), has been increasing in the Western ${ }^{1-3}$ and Asian countries ${ }^{4}$.

In South Korea, health care services are provided through primary and secondary care facility. While primary care services are provided through non-tertiary hospital (clinics, hospitals, and general hospitals), patients can access secondary care through tertiary hospitals (advanced general hospitals) ${ }^{5}$. The health care delivery system is introduced to utilize medical resources efficiently (to limit patients' herd behavior to seek tertiary hospital services), establish the roles of medical institutions, and help curb the rise in national medical expenditures and to secure financial sustainability ${ }^{6}$. However, patients themselves can choose their medical providers, giving them access to medical institutions without too many restrictions. With no strict gatekeeping system, it is relatively easy for patients to access secondary care in tertiary hospitals. In addition, patients generally prefer tertiary hospitals to non-tertiary hospitals because they believe that the quality of care that is provided by tertiary hospitals is better than that of non-tertiary hospitals ${ }^{7}$. These engender the concentration of patients in tertiary hospitals and results in the waste of health care resource and delay of timely treatment ${ }^{8}$. This tendency is also observed in

\footnotetext{
${ }^{1}$ Department of Biostatistics, College of Medicine, Korea University, Seoul, Korea. ${ }^{2}$ Department of Cardiology, Ulsan University Hospital, University of Ulsan College of Medicine, Ulsan, Korea. ${ }^{3}$ Division of Cardiology, Pusan National University Yangsan Hospital, Pusan National University of Medicine, Yangsan, Korea. ${ }^{4}$ Division of Cardiology, Department of Internal Medicine, Konkuk University Medical Center, Konkuk University School of Medicine, Seoul, Korea. ${ }^{5}$ Division of Cardiology, Chungnam National University Sejong Hospital, Chungnam National University School of Medicine, Sejong, Korea. ${ }^{\square}$ email: apollo0822@naver.com
} 
CIED procedures. Although the number of tertiary hospitals was only about a quarter of total number of medical institutions that performed pacemaker procedure, more than $60 \%$ of pacemaker procedures were performed in tertiary hospitals 9 . However, there is paucity of data comparing the clinical outcomes of CIED procedures between tertiary hospitals and non-tertiary hospitals. Therefore, we aimed to compare the characteristics and mortality of new implantation of CIED between tertiary hospitals and non-tertiary hospitals.

\section{Methods}

Data sources. In South Korea, all healthcare providers had to join the national health insurance (NHI) system on a fee-for-service basis. The Health Insurance Review \& Assessment Service (HIRA) is a quasi-governmental organization that systematically reviews medical fees to minimize the risk of redundant and unnecessary medical services. Thus, all NHI claims are reviewed by the HIRA ${ }^{10}$. For this study, data from 2013 to 2017 claims records of the HIRA were used. Patient information was anonymized and de-identified in the claims database of the HIRA. Diagnosis codes were used according to the International Classification of Diseases, 10th Revision (ICD-10). In addition, specific information about the procedure, devices, and drugs were identified by codes from the HIRA database ${ }^{10}$. This study was approved by the Institutional Review Board at Ulsan University Hospital, Ulsan, Korea. The requirement for informed consent was waived by the Institutional Review Board at Ulsan University Hospital because of the anonymity of the patients and the nonintrusive nature of the study. All methods were performed in accordance with the relevant guidelines and regulations.

Study population. From the claims database of the HIRA between July 2013 and June 2017, we identified patients who had undergone implantation of CIED (such as PPM, CRT-P, ICD, and CRT-D) and their corresponding device codes. To examine patients with first and new implantation of CIED, we excluded patients with generator change, generator removal, upgrade of CIED, and revision of leads. We also excluded patients with insufficient information of CIED type $(n=36)$ in the HIRA database. To identify the comorbid condition of patients, we selected those with at least 6 months of information prior to the index day. Furthermore, patients were categorized as PPM group (including PPM or CRT-P) and ICD group (including single/dual chamber ICD or CRT-D).

Then, patients were classified into the tertiary hospital group and non-tertiary hospital group according to the hospital where CIED procedures were performed. In the present study, the definition of tertiary hospitals is advanced general hospitals that were selected and authorized by the Ministry of Health and Welfare of South Korea during the study period $(2013-2017)^{11}$.

Study variables. The ICD-10 codes were used to identify comorbid conditions such as diabetes, diabetes with chronic complications, dyslipidemia, hypertension, congestive heart failure, peripheral vascular disease, cerebrovascular disease, chronic pulmonary disease, moderate to severe liver disease, and renal disease. The codes also identified cancer, rheumatic disease, atrial fibrillation, ventricular tachyarrhythmia (ventricular tachycardia [ICD-10 codes I47.0, and I47.2], ventricular fibrillation or flutter [ICD-10 codes I49.0]), and aborted cardiac arrest (ICD-10 codes I46.X) within 6 months prior to the index day ${ }^{10,12,13}$. The Charlson comorbidity index was obtained from the ICD-10 $\operatorname{codes}^{10}$. In the HIRA database, all prescribed medications were recorded with rigorous accuracy. Patients were considered to have hypertension, diabetes, and dyslipidemia if anti-hypertensive, anti-diabetic, and anti-dyslipidemic drugs were identified from the medication codes within 6 months prior to the index day ${ }^{10,14}$. For ICD implantation, ventricular tachyarrhythmia and aborted cardiac arrest within 6 months prior to the index day or at the index hospitalization were classified as indications for secondary prevention and the rest were classified as indications for primary prevention.

Clinical outcomes. All-cause death was identified by all in- and out-patient claims that indicated death. Inhospital death was defined as death occurred during the index hospitalization. In this study, for the evaluation of clinical outcomes, the HIRA database was used until December 2017.

Statistical analysis. All baseline patient characteristics and comorbid conditions were summarized as mean \pm standard deviation or frequency (percentage) for continuous or categorical variables, respectively. We evaluated whether there are differences for in-hospital mortality and all-cause death rates between tertiary and non-tertiary hospital group. We conducted separate analyses of the PPM and ICD groups. We also conducted subgroup analyses for ICD group according to subtypes of ICD (single/dual chamber ICD and CRT-D). Baseline patient characteristics were compared between tertiary and non-tertiary hospital group using the two sample T-test or the Fisher's exact test. For the in-hospital mortality, we used the logistic regression model, while we used the Cox proportional hazards regression model for the all-cause mortality rate analysis. In all regression analyses, the reference was the non-tertiary hospital group. We employed the propensity-score matching analysis to reduce the impact of potential confounding effects on the mortality risk comparison. The propensity-scores were derived nonparametrically using the variables of age, gender, hypertension, diabetes, diabetes with chronic complications, dyslipidemia, congestive heart failure, peripheral vascular disease, cerebrovascular disease, chronic pulmonary disease, moderate to severe liver disease, renal disease, cancer, rheumatic disease, atrial fibrillation, ventricular tachyarrhythmia, aborted cardiac arrest, Charlson comorbidity index, type of CIED, and indication of ICD. We used the nearest neighbor matching approach with a caliper size of 0.2 and evaluated the matching quality by measuring the covariate balancing in the matched set. We computed standardized differences in means between the two groups and considered the covariate balance achieved as long as the absolute standardized difference is less than 0.2. All standardized differences in the covariates were less than 0.05 . To account for the matched pairs, we used the generalized estimating equations for the in-hospital mortality, as well as the Cox 


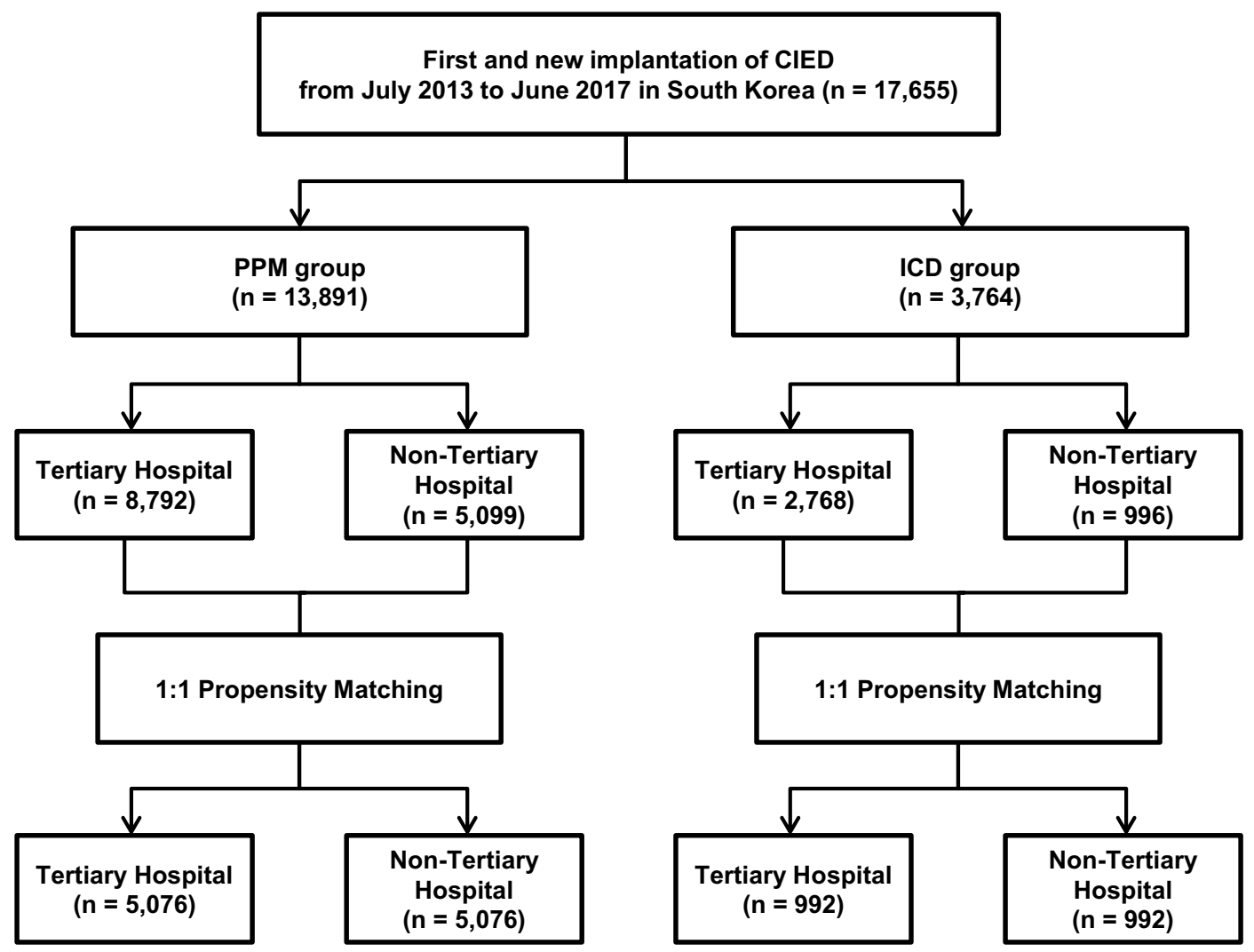

Figure 1. Diagrammatic representation of the study. CIED, cardiac implantable electronic device; ICD, implantable cardioverter-defibrillator; PPM, permanent pacemaker.

regression model with the sandwich standard errors for time to event outcome of the all-cause mortality. All data analyses were performed using the R software version 3.6.3 (R Foundation for Statistical Computing, Vienna, Austria; www.r-project.org). R 'MatchIt' package was used for the propensity-score matching. A $p$-value $<0.05$ was considered significant for all two-sided tests.

\section{Results}

Study population and baseline characteristics. Between July 2013 and June 2017, a total of 17,655 patients undergoing first and new implantation of CIED were identified from the claims database of HIRA (Fig. 1). The mean age of study participants was $68.5 \pm 13.6$ years and 8596 (48.7\%) were male. Hypertension, diabetes, dyslipidemia, congestive heart failure, and atrial fibrillation were observed in 13,311 (75.4\%), 6310 (35.7\%), 10,268 (58.2\%), 5232 (29.6\%), and 4147 (23.5\%) patients, respectively. The annual number of CIED procedures was had significantly increased during the study period (Table 1). According to the hospital categories, 11,560 (65.5\%) patients were in the tertiary hospital group and 6095 (34.5\%) patients were in the nontertiary hospital group. The number of hospitals providing CIED (including PPM, CRT-P, ICD, or CRT-D) in the tertiary hospital group and non-tertiary hospital group were 47 and 127, respectively. Tertiary hospital group performed more CIED procedures $(61.9 \pm 53.4$ per year) than non-tertiary hospital group (11.9 \pm 19.0 per year).

Tertiary hospital versus non-tertiary hospital in PPM group. According to the CIED procedures, the study participants who underwent PPM or CRT-P implantation were classified as PPM group $(n=13,891)$. Among them, patients were categorized into the tertiary hospital group $(n=8792)$ and non-tertiary hospital group $(n=5099)$. Patients in non-tertiary hospital group were older and had more comorbidities than those in tertiary hospital group (Table 1). Tertiary hospital group performed more implantation of dual chamber and epicardial system pacemaker compare to non-tertiary hospital group.

In-hospital mortality of tertiary and non-tertiary hospital group was $0.4 \%$ and $0.6 \%$, respectively. There was no significant difference in the in-hospital mortality between tertiary and non-tertiary hospital group (unadjusted odds ratio $[\mathrm{OR}]$ of tertiary hospital: $0.68,95 \%$ confidence interval $[\mathrm{CI}]: 0.41-1.12, p=0.13$ ). During a mean follow-up of $2.2 \pm 1.2$ years, non-tertiary hospital group was more likely to have all-cause death than tertiary hospital group (unadjusted hazard ratio [HR] of tertiary hospital: 0.85, 95\% CI 0.76-0.96, $p=0.008$ ). Figure 2a shows the unadjusted cumulative incidence rates for all-cause deaths of the two groups.

After propensity-score matching, there were 5076 matched pairs. In the matched cohort, there were no other significant differences between tertiary and non-tertiary hospital group for any of the covariates (Table 2). There was no significant difference in terms of the incidence of in-hospital mortality between tertiary and non-tertiary 


\begin{tabular}{|c|c|c|c|c|c|c|}
\hline \multirow[b]{3}{*}{ Characteristics } & \multicolumn{3}{|c|}{ PPM group $(n=13,891)$} & \multicolumn{3}{|c|}{ ICD group $(n=3764)$} \\
\hline & \multirow{2}{*}{\begin{tabular}{|l|} 
Tertiary hospital \\
$(\mathbf{n}=\mathbf{8 7 9 2})$
\end{tabular}} & \multirow{2}{*}{\begin{tabular}{|l|} 
Non-tertiary hospital \\
$(n=5099)$ \\
\end{tabular}} & \multirow[b]{2}{*}{$p$ value } & \multirow{2}{*}{$\begin{array}{l}\text { Tertiary hospital } \\
(n=2768)\end{array}$} & \multirow{2}{*}{\begin{tabular}{|l|} 
Non-tertiary hospital \\
$(\mathrm{n}=996)$
\end{tabular}} & \multirow[b]{2}{*}{$p$ value } \\
\hline & & & & & & \\
\hline Enrolled number (\%) & & & 0.012 & & & 0.016 \\
\hline July 2013 to June 2014 & $1970(22.4 \%)$ & $1051(20.6 \%)$ & & $553(20.0 \%)$ & $180(18.1 \%)$ & \\
\hline July 2014 to June 2015 & $2096(23.8 \%)$ & $1168(22.9 \%)$ & & $696(25.1 \%)$ & $213(21.4 \%)$ & \\
\hline July 2015 to June 2016 & $2309(26.3 \%)$ & $1382(27.1 \%)$ & & $729(26.3 \%)$ & $279(28.0 \%)$ & \\
\hline July 2016 to June 2017 & $2417(27.5 \%)$ & $1498(29.4 \%)$ & & $790(28.5 \%)$ & $324(32.5 \%)$ & \\
\hline Age, years & $70.5 \pm 12.0$ & $72.9 \pm 10.7$ & $<0.001$ & $57.1 \pm 15.4$ & $60.5 \pm 14.6$ & $<0.001$ \\
\hline CIED rate by age category & & & $<0.001$ & & & $<0.001$ \\
\hline$<20$ & $18(0.2 \%)$ & $5(0.1 \%)$ & & $37(1.3 \%)$ & $3(0.3 \%)$ & \\
\hline $20-60$ & $1581(18.0 \%)$ & $653(12.8 \%)$ & & $1477(53.4 \%)$ & $442(44.4 \%)$ & \\
\hline $61-80$ & $5544(63.1 \%)$ & $3161(62.0 \%)$ & & $1170(42.3 \%)$ & $500(50.2 \%)$ & \\
\hline$>80$ & $1648(18.7 \%)$ & $1280(25.1 \%)$ & & $84(3.0 \%)$ & $51(5.1 \%)$ & \\
\hline Male (\%) & $3723(42.3 \%)$ & $2158(42.3 \%)$ & 0.99 & $1990(71.9 \%)$ & $725(72.8 \%)$ & 0.62 \\
\hline \multicolumn{7}{|l|}{ Comorbid conditions (\%) } \\
\hline Hypertension & $6538(74.4 \%)$ & $4052(79.5 \%)$ & $<0.001$ & $1968(71.1 \%)$ & $753(75.6 \%)$ & 0.006 \\
\hline Diabetes & $2971(33.8 \%)$ & $1929(37.8 \%)$ & $<0.001$ & $981(35.4 \%)$ & $405(40.7 \%)$ & 0.004 \\
\hline $\begin{array}{l}\text { Diabetes with chronic } \\
\text { complications }^{*}\end{array}$ & $8(0.1 \%)$ & $12(0.2 \%)$ & 0.04 & $2(0.1 \%)$ & $2(0.2 \%)$ & 0.29 \\
\hline Dyslipidemia & $4971(56.5 \%)$ & $3066(60.1 \%)$ & $<0.001$ & $1585(57.3 \%)$ & $646(64.9 \%)$ & $<0.001$ \\
\hline Congestive heart failure & $1894(21.5 \%)$ & $1218(23.9 \%)$ & 0.002 & $1521(54.9 \%)$ & $599(60.1 \%)$ & 0.005 \\
\hline $\begin{array}{l}\text { Peripheral vascular } \\
\text { disease }\end{array}$ & $1236(14.1 \%)$ & $807(15.8 \%)$ & 0.005 & $221(8.0 \%)$ & $77(7.7 \%)$ & 0.84 \\
\hline Cerebrovascular disease & $1877(21.3 \%)$ & $1218(23.9 \%)$ & 0.001 & $319(11.5 \%)$ & $131(13.2 \%)$ & 0.19 \\
\hline $\begin{array}{l}\text { Chronic pulmonary } \\
\text { disease }\end{array}$ & $1815(20.6 \%)$ & $1058(20.7 \%)$ & 0.90 & $538(19.4 \%)$ & $225(22.6 \%)$ & 0.04 \\
\hline $\begin{array}{l}\text { Moderate to severe liver } \\
\text { disease }\end{array}$ & $21(0.2 \%)$ & $12(0.2 \%)$ & $>0.99$ & $0(0.0 \%)$ & $0(0.0 \%)$ & N/A \\
\hline Renal disease & $660(7.5 \%)$ & $388(7.6 \%)$ & 0.84 & $259(9.4 \%)$ & $94(9.4 \%)$ & 0.95 \\
\hline Cancer & $303(3.4 \%)$ & $137(2.7 \%)$ & 0.01 & $57(2.1 \%)$ & $22(2.2 \%)$ & 0.80 \\
\hline Rheumatic disease & $9(0.1 \%)$ & $11(0.2 \%)$ & 0.11 & $12(0.4 \%)$ & $2(0.2 \%)$ & 0.38 \\
\hline Atrial fibrillation & $2273(25.9 \%)$ & $1091(21.4 \%)$ & $<0.001$ & $578(20.9 \%)$ & $205(20.6 \%)$ & 0.86 \\
\hline $\begin{array}{l}\text { Ventricular tachyar- } \\
\text { rhythmia }\end{array}$ & $126(1.4 \%)$ & $50(1.0 \%)$ & 0.02 & $537(19.4 \%)$ & $145(14.6 \%)$ & 0.001 \\
\hline Aborted cardiac arrest & $18(0.2 \%)$ & $16(0.3 \%)$ & 0.22 & $446(16.1 \%)$ & $113(11.3 \%)$ & $<0.001$ \\
\hline $\begin{array}{l}\text { Charlson comorbidity } \\
\text { index }\end{array}$ & $1.83 \pm 1.63$ & $1.94 \pm 1.63$ & $<0.001$ & $1.89 \pm 1.62$ & $2.12 \pm 1.77$ & 0.001 \\
\hline $\begin{array}{l}\text { Procedure }{ }^{\S} \text { volume of } \\
\text { hospital per year }\end{array}$ & $61.9 \pm 53.4$ & $11.9 \pm 19.0$ & $<0.001$ & $61.9 \pm 53.4$ & $11.9 \pm 19.0$ & $<0.001$ \\
\hline \multicolumn{7}{|l|}{ Type of CIED } \\
\hline \multicolumn{7}{|l|}{ PPM } \\
\hline Single chamber & $1290(14.7 \%)$ & $930(18.2 \%)$ & $<0.001$ & & & \\
\hline Dual chamber & $7404(84.2 \%)$ & $4146(81.3 \%)$ & $<0.001$ & & & \\
\hline CRT-P & $22(0.3 \%)$ & $12(0.2 \%)$ & $>0.99$ & & & \\
\hline Epicardial system & $76(0.9 \%)$ & $11(0.2 \%)$ & $<0.001$ & & & \\
\hline \multicolumn{7}{|l|}{ ICD } \\
\hline Single chamber & & & & $1305(47.1 \%)$ & $527(52.9 \%)$ & 0.002 \\
\hline Dual chamber & & & & $935(33.8 \%)$ & $276(27.7 \%)$ & $<0.001$ \\
\hline CRT-D & & & & $528(19.1 \%)$ & $193(19.4 \%)$ & 0.85 \\
\hline Indication of ICD & - & - & - & - & & $<0.001$ \\
\hline Primary prevention & & & & $1073(38.8 \%)$ & $451(45.3 \%)$ & \\
\hline Secondary prevention & - & - & - & $1695(61.2 \%)$ & $545(54.7 \%)$ & \\
\hline In-hospital mortality & $34(0.4 \%)$ & $29(0.6 \%)$ & 0.15 & $16(0.6 \%)$ & $4(0.4 \%)$ & 0.62 \\
\hline
\end{tabular}

Table 1. Characteristics of patients undergoing new implantation of cardiac implantable electronic device and of hospitals that performed new implantation of cardiac implantable electronic device in tertiary and non-tertiary hospital between 2013 and 2017. Data are reported as mean \pm SD or as number (\%). CIED, cardiac implantable electronic device; CRT-D, cardiac resynchronization therapy with defibrillator; CRT-P, cardiac resynchronization therapy with pacemaker; ICD, implantable cardioverter-defibrillator; PPM, permanent pacemaker. ${ }^{\star}$ Including diabetic nephropathy, retinopathy, or neuropathy. ${ }^{\S}$ First and new implantation of CIED including PPM, CRT-P, ICD, and CRT-D. 


\section{a}

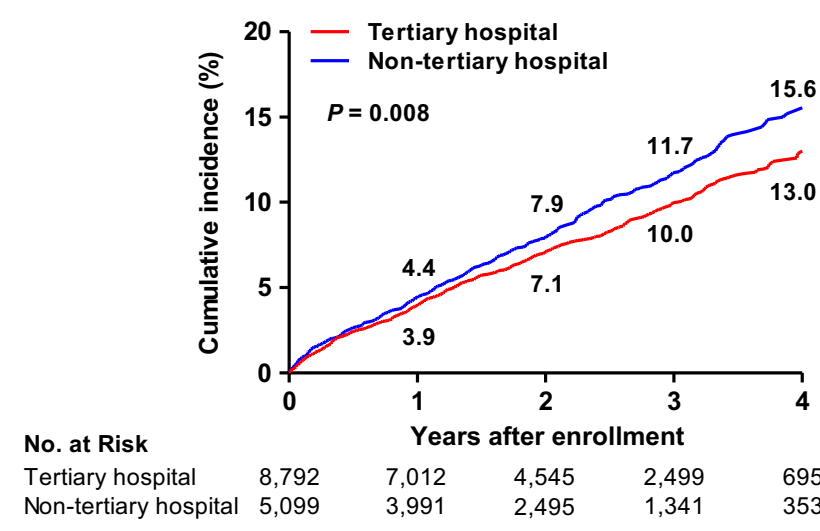

b

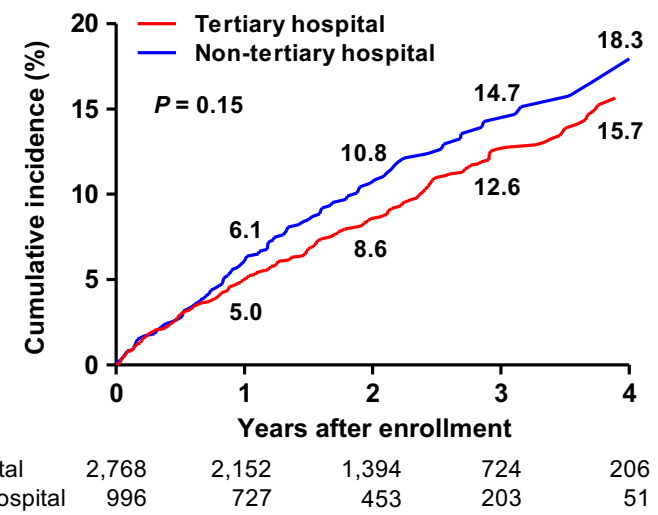

Figure 2. Unadjusted cumulative incidence rates for all-cause deaths in the study population. Cumulative incidence curves are shown for all-cause death in patients with PPM group (a) and in those with ICD group (b). The numbers in each figure represent the cumulative incidence rates at each time point. All $p$ values were calculated with the use of the log-rank test. ICD, implantable cardioverter-defibrillator; PPM, permanent pacemaker.

hospital group (OR of tertiary hospital: $0.76,95 \%$ CI $0.43-1.32, p=0.33$ ). During the follow-up period (mean, $2.1 \pm 1.2$ years), there was no difference in the incidence of all-cause death (HR of tertiary hospital: $0.92,95 \%$ CI $0.81-1.05, p=0.24$ ) between tertiary and non-tertiary hospital group (Table 3 ).

Tertiary hospital versus non-tertiary hospital in ICD group. We also analyzed the patients with ICD group including single/dual chamber ICD or CRT-D $(n=3764)$. Among them, patients were categorized into the tertiary hospital group $(n=2768)$ and non-tertiary hospital group $(n=996)$. Patients in non-tertiary hospital group were older and had more comorbidities than those in tertiary hospital group (Table 1). Tertiary hospital group performed more implantation of dual chamber ICD compare to non-tertiary hospital group. In addition, tertiary hospital group performed more implantation of ICD for secondary prevention compare to non-tertiary hospital group.

In-hospital mortality of tertiary and non-tertiary hospital group was $0.6 \%$ and $0.4 \%$, respectively. There was no significant difference in the in-hospital mortality between tertiary and non-tertiary hospital group (unadjusted OR of tertiary hospital: $1.44,95 \%$ CI $0.48-4.32, p=0.51$ ). During a mean follow-up of $2.1 \pm 1.2$ years, there was no significant difference in all-cause death between the two groups (unadjusted HR of tertiary hospital: 0.85 , $95 \%$ CI $0.67-1.06, p=0.15)$. Figure $2 \mathrm{~b}$ shows the unadjusted cumulative incidence rates for all-cause deaths of the two groups.

After propensity-score matching, there were 992 matched pairs. In the matched cohort, no significant differences were observed in terms of covariates between the two groups (Table 2). There was no significant difference in terms of the incidence of in-hospital mortality between the two groups (OR of tertiary hospital: 1.76, 95\% CI $0.51-6.02, p=0.37$ ). In addition, the incidence of all-cause death (HR of tertiary hospital: $0.95,95 \%$ CI $0.72-1.24$, $p=0.70$ ) was not different between tertiary and non-tertiary hospital group during the follow-up period (mean, $2.0 \pm 1.2$ years) (Table 3 ).

We also conducted subgroup analyses for ICD group according to subtypes of ICD (single/dual chamber ICD and CRT-D). Baseline characteristics of both ICD subtypes were shown in Supplementary Table S1 online. After propensity-score matching, there were no significant differences between tertiary and non-tertiary hospital group for any of the covariates in both ICD subtypes (Supplementary Table S2 online). In both subtypes of ICD, there were no significant differences in the incidence of in-hospital mortality and all-cause death between tertiary and non-tertiary hospital group (Supplementary Table S3 online).

\section{Discussion}

This study aimed to compare the characteristics and mortality of first and new implantation of CIED between tertiary and non-tertiary hospitals. The major findings of the present study using NHI claims data in South Korea are as follows: (1) patients in non-tertiary hospital group were older and had more comorbidities than those in tertiary hospital group; (2) after propensity-score matching, the incidences of in-hospital death and all-cause death were not significantly different between tertiary and non-tertiary hospitals in PPM and ICD group.

In this study, patients in non-tertiary hospitals were older and had more comorbidities compare to those in tertiary hospitals. This finding could be explained by the location of the tertiary (mostly urban region) and non-tertiary (mostly suburban and rural region) hospitals. In this study, $70.2 \%$ of tertiary hospitals were located in urban region. In Korea, the mean age of the population in suburban and rural region was higher than that in urban region and comorbidities (hypertension, diabetes, and congestive heart failure) were more prevalent in suburban and rural regions ${ }^{15}$. This difference between urban and rural region were also reported in previous studies $^{16-18}$ 


\begin{tabular}{|c|c|c|c|c|c|c|}
\hline \multirow[b]{3}{*}{ Characteristics } & \multicolumn{3}{|c|}{ PPM group ( $n=5076$ pairs) } & \multicolumn{3}{|c|}{ ICD group ( $n=992$ pairs) } \\
\hline & \multirow{2}{*}{\begin{tabular}{|l|} 
Tertiary hospital \\
$(\mathbf{n}=\mathbf{5 0 7 6})$ \\
\end{tabular}} & \multirow{2}{*}{$\begin{array}{l}\text { Non-tertiary hospital } \\
(n=5076)\end{array}$} & \multirow[b]{2}{*}{$p$ value } & \multirow{2}{*}{$\begin{array}{l}\text { Tertiary hospital } \\
(\mathrm{n}=992)\end{array}$} & \multirow{2}{*}{\begin{tabular}{|l|} 
Non-tertiary hospital \\
$(n=992)$
\end{tabular}} & \multirow[b]{2}{*}{$p$ value } \\
\hline & & & & & & \\
\hline Age, years & $72.8 \pm 10.8$ & $72.9 \pm 10.7$ & 0.90 & $60.2 \pm 14.0$ & $60.5 \pm 14.6$ & 0.16 \\
\hline Male (\%) & $2161(42.6 \%)$ & $2151(42.4 \%)$ & 0.95 & $729(73.5 \%)$ & $722(72.8 \%)$ & 0.62 \\
\hline \multicolumn{7}{|l|}{ Comorbid conditions (\%) } \\
\hline Hypertension & $4017(79.1 \%)$ & $4030(79.4 \%)$ & 0.18 & $740(74.6 \%)$ & $750(75.6 \%)$ & 0.26 \\
\hline Diabetes & $1904(37.5 \%)$ & $1914(37.7 \%)$ & 0.82 & $408(41.1 \%)$ & $402(40.5 \%)$ & 0.81 \\
\hline $\begin{array}{l}\text { Diabetes with chronic } \\
\text { complications* }\end{array}$ & $7(0.1 \%)$ & $8(0.2 \%)$ & 0.07 & $1(0.1 \%)$ & $2(0.2 \%)$ & $>0.99$ \\
\hline Dyslipidemia & $3009(59.3 \%)$ & $3045(60.0 \%)$ & 0.98 & $627(63.2 \%)$ & $643(64.8 \%)$ & 0.80 \\
\hline Congestive heart failure & $1191(23.5 \%)$ & $1205(23.7 \%)$ & 0.65 & $578(58.3 \%)$ & $596(60.1 \%)$ & 0.47 \\
\hline $\begin{array}{l}\text { Peripheral vascular } \\
\text { disease }\end{array}$ & $787(15.5 \%)$ & $798(15.7 \%)$ & 0.54 & $69(7.0 \%)$ & $77(7.8 \%)$ & 0.07 \\
\hline Cerebrovascular disease & $1200(23.6 \%)$ & $1206(23.8 \%)$ & 0.47 & $125(12.6 \%)$ & $131(13.2 \%)$ & 0.53 \\
\hline $\begin{array}{l}\text { Chronic pulmonary } \\
\text { disease }\end{array}$ & $1020(20.1 \%)$ & $1054(20.8 \%)$ & 0.90 & $225(22.7 \%)$ & $224(22.6 \%)$ & 0.83 \\
\hline $\begin{array}{l}\text { Moderate to severe liver } \\
\text { disease }\end{array}$ & $14(0.3 \%)$ & $11(0.2 \%)$ & 0.69 & $0(0.0 \%)$ & $0(0.0 \%)$ & $\mathrm{N} / \mathrm{A}$ \\
\hline Renal disease & $394(7.8 \%)$ & $386(7.6 \%)$ & 0.32 & $85(8.6 \%)$ & $94(9.5 \%)$ & 0.87 \\
\hline Cancer & $132(2.6 \%)$ & $137(2.7 \%)$ & 0.21 & $16(1.6 \%)$ & $18(1.8 \%)$ & 0.39 \\
\hline Rheumatic disease & $9(0.2 \%)$ & $9(0.2 \%)$ & 0.45 & $1(0.1 \%)$ & $2(0.2 \%)$ & $>0.99$ \\
\hline Atrial fibrillation & $1128(22.2 \%)$ & $1091(21.5 \%)$ & 0.77 & $192(19.4 \%)$ & $205(20.7 \%)$ & 0.44 \\
\hline $\begin{array}{l}\text { Ventricular tachyar- } \\
\text { rhythmia }\end{array}$ & $59(1.2 \%)$ & $50(1.0 \%)$ & 0.56 & $139(14.0 \%)$ & $145(14.6 \%)$ & 0.73 \\
\hline Aborted cardiac arrest & $15(0.3 \%)$ & $16(0.3 \%)$ & 0.72 & $114(11.5 \%)$ & $112(11.3 \%)$ & 0.59 \\
\hline $\begin{array}{l}\text { Charlson comorbidity } \\
\text { index }\end{array}$ & $1.91 \pm 1.62$ & $1.93 \pm 1.62$ & 0.59 & $2.05 \pm 1.63$ & $2.10 \pm 1.72$ & 0.71 \\
\hline \multicolumn{7}{|l|}{ Type of CIED } \\
\hline PPM & & & 0.40 & & & \\
\hline Single chamber & $897(17.7 \%)$ & $913(18.0 \%)$ & & - & - & \\
\hline Dual chamber & $4148(81.7 \%)$ & $4140(81.6 \%)$ & & - & - & \\
\hline $\begin{array}{l}\text { Epicardial system, } \\
\text { CRT-P }\end{array}$ & $31(0.6 \%)$ & $23(0.5 \%)$ & & - & - & \\
\hline ICD & & & & & & 0.59 \\
\hline Single chamber & - & - & & $508(51.2 \%)$ & $525(52.9 \%)$ & \\
\hline Dual chamber & - & - & & $276(27.8 \%)$ & $275(27.7 \%)$ & \\
\hline CRT-D & - & - & & $208(21.0 \%)$ & $192(19.4 \%)$ & \\
\hline Indication of ICD & - & - & & & & 0.41 \\
\hline Primary prevention & & & & $444(44.8 \%)$ & $448(45.2 \%)$ & \\
\hline Secondary prevention & - & - & & $548(55.2 \%)$ & $544(54.8 \%)$ & \\
\hline
\end{tabular}

Table 2. Baseline characteristics of the propensity-score matched patients undergoing new implantation of cardiac implantable electronic device in tertiary and non-tertiary hospital. Data are reported as mean \pm SD or as number (\%). CIED, cardiac implantable electronic device; CRT-D, cardiac resynchronization therapy with defibrillator; CRT-P, cardiac resynchronization therapy with pacemaker; ICD, implantable cardioverterdefibrillator; PPM, permanent pacemaker. ${ }^{\star}$ Including diabetic nephropathy, retinopathy, or neuropathy.

\begin{tabular}{|l|l|l|l|l|l|}
\hline \multirow{2}{*}{ Propensity-score matched analysis } & \multicolumn{4}{l|}{ PPM group $(\mathbf{n}=\mathbf{5 0 7 6}$ pairs) } & \multicolumn{2}{l|}{ ICD group (n=992 pairs) } \\
\cline { 2 - 5 } & Tertiary hospital compared to non-tertiary hospital \\
\hline & Odds ratio $(95 \% \mathrm{CI})$ & $p$ value & Odds ratio $(95 \% \mathrm{CI})$ & $p$ value \\
\hline In-hospital mortality & $0.76(0.43-1.32)$ & 0.33 & $1.76(0.51-6.02)$ & 0.37 \\
\hline & Hazard ratio $(95 \% \mathrm{CI})$ & $p$ value & Hazard ratio $(95 \% \mathrm{CI})$ & $p$ value \\
\hline All-cause death & $0.92(0.81-1.05)$ & 0.24 & $0.95(0.72-1.24)$ & 0.70 \\
\hline
\end{tabular}

Table 3. Propensity score matched in-hospital mortality and all-cause death of patients undergoing new implantation of cardiac implantable electronic device in tertiary and non-tertiary hospital. CI, confidence interval; ICD, implantable cardioverter-defibrillator; PPM, permanent pacemaker. 
Tertiary hospital is a medical facility which provides a high degree of subspecialty expertise for patients ${ }^{19}$ and is generally larger and provide more procedure compare to non-tertiary hospital. Consequently, tertiary hospital performed more CIED procedure than non-tertiary hospital (62 versus 12 per year) in this study. Several previous studies demonstrated that high procedure volume hospital is less likely to have an adverse outcome after CIED implantation. Nowak et al. reported that hospital annual PPM volume was inversely related to surgical complications and atrial or ventricular dislocation ${ }^{20}$. Similar trends were also observed in $\mathrm{ICD}^{21}$. Unlike previous studies, however, we did not observe a better prognosis among patients in the tertiary hospital, high procedure volume hospital, compare to non-tertiary hospital. This might be explained by the difference in the definition of the study outcomes compared to previous studies. Most previous studies defined the study outcomes to include not only death but also traumatic complications (pneumothorax, hemothorax, pericardial effusion, and pericardial tamponade), lead-related complications, and device-related infection ${ }^{20,21}$. When the definition of study outcome was confined to mortality, however, procedure volume did not have a predictive value. Freeman et al. reported that adverse events (including cardiac arrest, cardiac perforation, pneumothorax, hemothorax, lead dislodgement, and device-related infection) were significantly higher in the lowest-procedure volume quartile compared to the highest-procedure volume quartile (OR 1.26, 95\% CI 1.05-1.52, $p<0.0001)^{21}$. However, in-hospital death was not different according to procedure volume. Another study also did not show a consistent procedure volumemortality relationship ${ }^{22}$. In addition to procedure volume, tertiary hospitals are teaching hospital and have more medical personnel, facility, and equipment compared to non-tertiary hospitals because designation criteria for tertiary hospitals are consist of these hospital characteristics ${ }^{11}$. However, we did not observe a better prognosis among patients in the tertiary hospital compare to non-tertiary hospital. This is consistent with previous study ${ }^{22}$.

Several previous studies have shown that operator characteristics are associated with clinical outcomes. AlKhatib et al. showed that although there was no correlation between operator volume of ICD implantation and 90-day mortality, 90-day rates of mechanical complication (OR 1.47, 95\% CI 1.09-1.99) and ICD infection (OR 2.47, 95\% CI 1.18-5.17) were significantly higher among operator in the lowest volume quartile compared with those in the highest volume quartile ${ }^{23}$. Curtis et al. reported that ICD implantations by a non-electrophysiologist were associated with a higher risk of in-hospital procedural complications compared to ICD implantation by an electrophysiologist ${ }^{24}$. Our study did not capture the risk associated with the operator characteristics. Therefore, further clinical studies are needed to evaluate it.

Concentration of patients in tertiary hospitals causes not only the economic problem in terms of waste of health care resource and the loss of opportunity cost for other hospitals but also the delay of timely treatment. Previous studies showed that patients tend to make choices based on hospital size, facility, modernity, and professional credibility ${ }^{7,8}$. The phenomenon of the concentration in tertiary hospitals might derive due to the lack of information (such as clinical outcomes). The results of the present study could be helpful for patients' decision to choose the hospitals and might alleviate the concentration of patients to tertiary hospitals in CIED procedures.

This study had several limitations. First, this study was a retrospective, observational study. Although we rigorously adjusted for baseline covariates using propensity-score matching, there are inherent limitations of a non-randomized study. Second, our study was based on administrative data from the HIRA in South Korea. In patients with CIED, procedural and device-related complications, such as traumatic-, lead-related complications, lead dislodgement, and device-related infection, are important clinical outcomes in addition to mortality. Similar to previous study using administrative databases ${ }^{22}$, this study could not identify these specific types of complications. In addition, we did not specify the cause of death. However, mortality is the most powerful hard endpoint and unbiased endpoint. Furthermore, to the best of our knowledge, this study is the largest study for mortality in Asian patients undergoing first and new implantation of CIED. Finally, this study only included the Korean population, and this may limit the applicability of our findings to other countries. However, considering the paucity of data concerning Asian populations, we believe that this study may have clinical implications.

In conclusion, this nationwide study suggested that mortality was not different between tertiary and non-tertiary hospitals in Korean patients undergoing first and new implantation of CIED. Further prospective, national cohort studies are needed to confirm these findings.

\section{Data availability}

The present study analyzed the NHI claims data in South Korea. Data of the NHI claims are accessible to researchers after permission of the HIRA in South Korea. Qualified, interested researchers may request access to these data from the HIRA (http://opendata.hira.or.kr/home.do). The authors do not have any special access privileges to these data.

Received: 1 August 2020; Accepted: 28 January 2021

Published online: 15 March 2021

\section{References}

1. Uslan, D. Z. et al. Temporal trends in permanent pacemaker implantation: a population-based study. Am. Heart J. 155, 896-903 (2008).

2. Kurtz, S. M. et al. Implantation trends and patient profiles for pacemakers and implantable cardioverter defibrillators in the United States: 1993-2006. Pacing Clin. Electrophysiol. 33, 705-711 (2010).

3. Mond, H. G. \& Proclemer, A. The 11th world survey of cardiac pacing and implantable cardioverter-defibrillators: calendar year 2009-a World Society of Arrhythmia's project. Pacing Clin. Electrophysiol. 34, 1013-1027 (2011).

4. Asia Pacific Heart Rhythm Society. APHRS White Book 2016 http://www.aphrs.org/attachments/article/42/APHRS\%20White\%20 Book\%202016.pdf (2016).

5. Chun, C., Kim, S. J. L. \& Lee, S. Republic of Korea: health system review. Health Syst. Transit. 11, 1-184 (2009).

6. National Health Insurance Service. Health Care Delivery System https://www.nhis.or.kr/static/html/wbd/g/a/wbdga0402.html (2010). 
7. Jung, H.-M. et al. The primary care performance of three types of medical institutions: a public survey using the Korean Primary Care Assessment Tool. Qual. Improv. Health Care 25, 16-25 (2019).

8. du Hong, P. \& Song, J. The effective distribution system for the concentration of patients to extra-large hospitals. J. Korean Surg. Soc. 80, 373-383 (2011).

9. National Health Insurance Service. Main Surgery Statistical Yearbook for 2017 https://www.gov.kr/portal/gvrnReport/view/G1812 000000007845? policyType=G00302\&srchTx (2017).

10. Han, S. et al. Trends, characteristics, and clinical outcomes of patients undergoing percutaneous coronary intervention in Korea between 2011 and 2015. Korean Circ. J. 48, 310-321 (2018).

11. Ministry of Health and Welfare. Designation criteria of advanced general hospital. http://www.mohw.go.kr/react/policy/index .jsp?PAR_MENU_ID=06\&MENU_ID=06290304\&PAGE=4\&topTitle (2019).

12. Han, S. et al. Effect of atrial fibrillation in Asian patients undergoing percutaneous coronary intervention with drug-eluting stents for stable coronary artery disease: results from a Korean nationwide study. Medicine (Baltimore) 97, e13488. https://doi.org/10.1097/ MD.0000000000013488 (2018).

13. Quan, H. et al. Coding algorithms for defining comorbidities in ICD-9-CM and ICD-10 administrative data. Med. Care 43, $1130-1139$ (2005).

14. Yang, Y. et al. Impact of diabetes mellitus in patients undergoing contemporary percutaneous coronary intervention: results from a Korean nationwide study. PLoS ONE 13, e0208746. https://doi.org/10.1371/journal.pone.0208746 (2018).

15. Lee, S. R., Choi, E. K., Han, K., Cha, M. J. \& Oh, S. Prevalence of non-valvular atrial fibrillation based on geographical distribution and socioeconomic status in the entire Korean population. Korean Circ. J. 48, 622-634 (2018).

16. O'Connor, A. \& Wellenius, G. Rural-urban disparities in the prevalence of diabetes and coronary heart disease. Public Health 126, $813-820(2012)$.

17. Abdul-Razak, S. et al. Prevalence, awareness, treatment, control and socio demographic determinants of hypertension in Malaysian adults. BMC Public Health 16, 351. https://doi.org/10.1186/s12889-016-3008-y (2016).

18. Wang, J. et al. Differences in prevalence of hypertension and associated risk factors in urban and rural residents of the northeastern region of the People's Republic of China: a cross-sectional study. PLoS ONE 13, e0195340. https://doi.org/10.1371/journ al.pone.0195340 (2018).

19. National Center for Biotechnology Information. Tertiary care centers https://www.ncbi.nlm.nih.gov/mesh/68062606 (2013).

20. Nowak, B. et al. Association between hospital procedure volume and early complications after pacemaker implantation: results from a large, unselected, contemporary cohort of the German nationwide obligatory external quality assurance programme. Europace 17, 787-793 (2015).

21. Freeman, J. V., Wang, Y., Curtis, J. P., Heidenreich, P. A. \& Hlatky, M. A. The relation between hospital procedure volume and complications of cardioverter-defibrillator implantation from the implantable cardioverter-defibrillator registry. J. Am. Coll. Cardiol. 56, 1133-1139 (2010).

22. Zhan, C., Baine, W. B., Sedrakyan, A. \& Steiner, C. Cardiac device implantation in the United States from 1997 through 2004: a population-based analysis. J. Gen. Intern. Med. 23(Suppl 1), 13-19 (2008).

23. Al-Khatib, S. M., Lucas, F. L., Jollis, J. G., Malenka, D. J. \& Wennberg, D. E. The relation between patients' outcomes and the volume of cardioverter-defibrillator implantation procedures performed by physicians treating Medicare beneficiaries. J. Am. Coll. Cardiol. 46, 1536-1540 (2005).

24. Curtis, J. P. et al. Association of physician certification and outcomes among patients receiving an implantable cardioverterdefibrillator. JAMA 301, 1661-1670 (2009).

\section{Acknowledgements}

This research was supported by Basic Science Research Program through the National Research Foundation of Korea funded by the Ministry of Education (2018R1D1A3B07043344) and the Ministry of Science, ICT \& Future Planning (2017R1C1B1006717).

\section{Author's contributions}

G.P. and Y.K. contributed to conception and design of study design. S.H., G.P., and Y.K. contributed to acquisition and analysis of data. S.H. and Y.K. contributed to interpretation of data and drafted the manuscript. K.W.H., C.H.K., and J.R. contributed to interpretation of data. S.P., K.W., S.H.A., S.K., and S.L. substantively revised the manuscript. All authors reviewed and approved the final manuscript.

\section{Competing interests}

The authors declare no competing interests.

\section{Additional information}

Supplementary Information The online version contains supplementary material available at https://doi. org/10.1038/s41598-021-83160-w.

Correspondence and requests for materials should be addressed to Y.-G.K.

Reprints and permissions information is available at www.nature.com/reprints.

Publisher's note Springer Nature remains neutral with regard to jurisdictional claims in published maps and institutional affiliations.

Open Access This article is licensed under a Creative Commons Attribution 4.0 International License, which permits use, sharing, adaptation, distribution and reproduction in any medium or format, as long as you give appropriate credit to the original author(s) and the source, provide a link to the Creative Commons licence, and indicate if changes were made. The images or other third party material in this article are included in the article's Creative Commons licence, unless indicated otherwise in a credit line to the material. If material is not included in the article's Creative Commons licence and your intended use is not permitted by statutory regulation or exceeds the permitted use, you will need to obtain permission directly from the copyright holder. To view a copy of this licence, visit http://creativecommons.org/licenses/by/4.0/.

(C) The Author(s) 2021 